\title{
Affordable evaluation of low frequency electric fields from the standpoint of Directive 2013/35/EU
}

\author{
Alexandru Salceanu, Eduard Lunca, Marius Paulet \\ "Gheorghe Asachi" Technical University of Iasi, Mangeron Str. 67, 700050, Iasi, Romania
}

\begin{abstract}
Since the 1st of July 2016, the Directive 2013/35/ EU on the employees' health protection in terms of non-ionizing electromagnetic radiations acquired the force of law.

An accessible methodology for the characterization of a workspace is proposed here, in terms of exposure to low-frequency electric fields.

Firstly, the means whereby an external electric field can induce electrical processes in the human body are presented, followed by a comparative summary of differently expressed exposure levels (ICNIRP, European Directive, IEEE-ICES).

Further on two electric field sensors are presented that can easily be hand-crafted in any laboratory, useful for extending the capabilities of a budget, low-frequency handheld spectrum analyzer. A realistic exposure metric is developed that cumulates the influence of all E-fields in the environment. A case study is presented on the cumulative assessment of exposure to low frequency electric fields produced in a laboratory-class where a network of 16 computers was working.

A simple numerical approach based on FEMM 4.2 has also been developed, for evaluating the E-field produced by overhead high voltage transmission lines.

This paper is an extended version of the original contribution to the IMEKO TC 42016 symposium in Budapest, Hungary.
\end{abstract}

Section: RESEARCH PAPER

Keywords: Directive 2013/35/EU; low frequency electric fields; health and safety

Citation: Alexandru Salceanu, Eduard Lunca, Marius Paulet, Affordable evaluation of low frequency electric fields from the standpoint of Directive 2013/35/EU, Acta IMEKO, vol. 6, no. 4, article 6, December 2017, identifier: IMEKO-ACTA-06 (2017)-04-06

Editor: Paul Regtien, Measurement Science Consultancy, The Netherlands

Received June 23, 2017; In final form November3, 2017; Published December 2017

Copyright: (C) 2017 IMEKO. This is an open-access article distributed under the terms of the Creative Commons Attribution 3.0 License, which permits unrestricted use, distribution, and reproduction in any medium, provided the original author and source are credited

Corresponding author: Alexandru Salceanu, e-mail: asalcean@tuiasi.ro

\section{CHALLENGES FOR ENGINEERS EMERGING FROM DIRECTIVE 2013/35/EU}

On the 26th of June 2013, in the Official Journal of the European Union, the Directive 2013/35/EU of The European Parliament and of The Council [1] was published, focused on the minimum health and safety requirements regarding the exposure of workers to electromagnetic fields.

This Directive already entered into force on the 1st of July 2016, being mandatory for any enterprise in the European Union to apply and comply with its provisions. Consequently, there is a large concern between employers, stakeholders and authorities regarding the effective application of the associated settlements. How could be decided that we have to deal or not with a dangerous situation? For the very beginning, it is the responsibility of the employer, but in the second phase, a state protection authority should check the orderliness and the conformation to the imposed limits. Basically it is about health and safety at every workplace, a fundamental conditioning all over Europe.

Even since June 1989, the European Union (EEC at that time) has unequivocally assumed the priorities in the field of employees' health and safety, through the adoption of the Framework Directive 391 on improving the conditions for the general protection of employees at work, the so called "Occupational Safety and Health" (OSH). Within this general Directive, the Directive 2004/40/EC, the 20-th specific one, has been also adopted, strictly focused on the risks arising from human exposure to electric and magnetic fields.

Due to the impressive growth rate of electromagnetic traffic and the quasi-generalized presence of equipment that uses either electricity or transmits information or energy through 
radio waves, the interest of both the general public and the specialists or authorities has increased in view of the potential negative effects on human health. This required the replacement of the above-mentioned Directive only after 9 years of applicability, which is a record in the "world" of European legislation.

This unusual dynamic is a convincing and plain argument for the actuality but also for the subtleties of the addressed issue. More specifically, the new Directive assumes more rigorous defined values for the so-called Exposure Limit Values (ELVs) and Action Levels (ALs) as regarding the induced electric fields in the body and respectively, the measured non-ionizing field strengths in the controlled environment.

In essence, this document does not deal with the possible long-term effects of variable electric and magnetic fields, a domain in which fundamentally different views and approaches exist in the international community of the strongest interdisciplinary collectives of researchers.

The Directive mainly establishes in an engineering manner the limitation of exposure to electrical and magnetic fields at values below those at which biophysical (direct or indirect) effects were scientifically proven.

One basic principle stipulates: better to prevent than to attempt restoring health! The original guidelines established by International Commission on Non-Ionizing Radiation Protection (ICNIRP) are based on the recording of short-term sensory effects related to action of internal electric fields on the central nervous system, the most well-known phenomenon being the "retinal phosphenes".

There are many (quasi-different) approaches regarding the "correlation" between the electric and magnetic field strengths in the human's environment and the induced electric fields and current densities in the human body.

Obviously, they are considered as unacceptable, current densities that become comparable to currents produced by heart beats or producing excitations of the optic or acoustic nerve.

Any employer should adopt actions aiming to protect his employees, by not overcoming these (measurable) values for the ALs of the electric and magnetic fields in the workplace neighborhood. The ELVs and ALs, stipulated in this Directive, are founded on the suggestions released by ICNIRP, in 1998 and later in 2010, even if the terms and the limit themselves are quite different.

More specifically, the ELVs refer to the maximum levels accepted for the electric fields induced in the human body due to the presence in the workers' environment of any alternative magnetic or electric fields, corresponding to the ICNIRP basic restrictions, while ALs represent the corresponding "ICNIRP reference values" for the external electric and magnetic fields that could be directly measured.

The effects might be: direct (thermal, mainly heating but also non-thermal, like electric fields induced in the body determining stimulation of muscles, nerves or sensory organs or like currents established in the limbs) or indirect, caused by the action of a specific object placed in the field (pacemakers, other implants, detonators or fires produced by sparks).

Stake-holders' expectations in relation to the part of the verification and control that must be done by the engineers concern first of all the correct measurement of the incident fields and, in the next stage, the calculation, analytical or by modeling, of the current densities or electric fields induced within the human body.

\section{SUPPORT FOR MICRO, SMALL AND MEDIUM-SIZED ENTERPRISES}

The Directive in its second chapter, entitled "Obligations of Employers" is executive, with three consecutive types of action: the assessment of risks, (identification of places with possible high exposure levels followed by their determination, by measurement or calculation), actions that should be done in order to avoid or at least to reduce the assessed risks and the effective participation of the workers at miscellaneous programs of training, also including continuous information and consultation.

For the effective implementation of Directive 2013/35/EU, the Commission has elaborated various practical guides, accepted by the regulation authorities in the domain that should provide a reliable scientific approach of the effective exposure to non-ionizing radiations, coupled with the evaluation of the efficiency of the adopted reducing methods [2].

There are two fundamental aspects that this Directive wants to regulate. Of course, mainly it is the health and safety of all EU workers. But equally important is the creation of a nondiscriminatory business environment which, by imposing the same restrictions on all (industrial) operators, supports the framework of a fair economic competition.

Is there really a need for technical and logistic support for Micro, Small and Medium-sized Enterprises (MSMEs)? Undoubtedly YES, the answer being argued by the following statistics.

In the EU, over $92 \%$ of enterprises are micro or small (having less than 10, respectively 50 employees), providing about $67 \%$ of jobs, thus being vital for the EU economy. In order to survive and withstand the market, they have to face many challenges, one of them being the compliance of the same quasi-tough restrictions from the point of view of OSH as the large enterprises, which have completely different human and financial resources.

Evidences of European Agency for Safety and Health at Work (EU-OSHA) show that MSMEs have greater difficulties in controlling risks than larger ones. Various studies, including the EU-OSHA's own European Survey of Enterprises on New and Emerging Risks (ESENER) [3], show that the challenges in handling OSH are particularly significant as the enterprises get smaller.

In other words, it is expectable as a medium or small employer cannot afford to permanently engage high qualified workforce, needing expensive instrumentation and sophisticated software for a reliable certification of the adherence to the stipulated exposure limits. Pre-compliance tests and methodologies should be easier to be performed, offering a good preparation for the strictly specialized measurements. The main objective of the here presented paper is to propose a practicable sequence and an affordable device for assessing the low frequency (up to $100 \mathrm{kHz}$ ) electric fields (LFEF) in the residential or occupational environment. Additionally, a modeling done by using open source software, which allows comparison with effectively performed measurements, leading to approximately equivalent or just only comparable results should be beneficial for validation. 


\section{E-FIELD EXPOSURE, FROM ELV TO AL}

The guidelines evolved by international authorities, mainly focused on health effects of the electric and magnetic fields, generally consider three "frequency milestones", $1 \mathrm{~Hz}, 100 \mathrm{kHz}$ and $10 \mathrm{MHz}$ that divide the spectrum in four ranges: static, low frequency, medium frequency and high frequency. This partition is justified by the intrinsic nature of the dominant effects: non-thermal (nerve or muscle stimulation), dominant for frequencies lower than $100 \mathrm{kHz}$ or thermal (heating of the tissue as a result of energy absorption, firstly in the skin region), for higher frequencies, with different measurement techniques and associated instrumentation used for an accurate determination.

For frequencies up to $100 \mathrm{kHz}$, the near field -far field border is at least at $500 \mathrm{~m}$ distance from the source, involving the compulsoriness of different measurements for the separated electric and respective magnetic fields [4].

In principle, high voltages (meaning high linear density of charge, measured in $\mathrm{C} / \mathrm{m}$ ) are generating dominant electric fields, while large currents are mainly sources of dominant magnetic fields. Obviously, the electric or magnetic fields have different units of measurement, so they cannot be compared as such. They could, however, be compared by means of the energy values (volumetric density) associated with them, $\varepsilon \mathrm{E}^{2} / 2$ and respective $\mu \mathrm{H}^{2} / 2$. If the densities of these stored energies are equal (in free space) the value $120 \pi(377 \Omega)$ is obtained for the ratio between electric and magnetic field strengths, the wellknown characteristic impedance of free space. A higher value for this ratio means the preponderance of the electric field.

By establishing the need for separate approaches, in this paper we only deal with human exposure to electric fields having frequencies lower than $100 \mathrm{kHz}$.

The electric fields are generated by the electric charges that produce a potential difference between the "plates" of a permanent or just occasionally appeared capacitor. The biunivocal link between voltage and electric field could be very easy disturbed by any object with some electric properties placed between the source and the measuring point. More specifically, the human body, a good conductor at low frequencies, significantly disturbs the distribution of the electric field lines in its vicinity.

There are many international agencies and organizations involved in establishing exposure restrictions on scientific basis: ICNIRP [5], [6], Institute of Electrical and Electronics Engineers-International Committee on Electromagnetic Safety (IEEE-ICES) [7], [8], World Health Organization (WHO) or International Agency for Research on Cancer (IARC).

An external (alternative) electric field induces a superficial electric charge on the surface of the exposed body. This phenomenon implies the emergence of internal currents

Table1. Health and sensory effects ELVs for internal electric field strengths (base restrictions); $f$ is the frequency expressed in hertz $(\mathrm{Hz})$.

\begin{tabular}{|c|c|c|}
\hline $\begin{array}{c}\text { Frequency range } \\
(\mathbf{H z})\end{array}$ & $\begin{array}{c}\text { Sensory effects ELVs, } \\
\text { peak values } \mathbf{( V / m )}\end{array}$ & $\begin{array}{c}\text { Health effects ELVs, } \\
\text { Peak values } \mathbf{( V / m})\end{array}$ \\
\hline $1-10$ & $0.7 / f$ & 1.1 \\
\hline $10-25$ & 0.07 & 1.1 \\
\hline $25-400$ & $0.0028 \times f$ & 1.1 \\
\hline $400-1000$ & $0.0028 \times f$ & 1.1 \\
\hline $10^{3}-3 \times 10^{3}$ & - & 1.1 \\
\hline $3 \times 10^{3}-10^{7}$ & - & $3.8 \times 10^{-4} \times f$ \\
\hline
\end{tabular}

required to "move" the induced electric charges on the body surface.

Physicians and biologists have ascertained internal electric fields with the smallest sensory effects and respective higher values at which adverse health effects may occur [1], Table 1.

It should be here recalled that in the previous documents elaborated by ICNIRP in 1998 [5] and assumed by EU immediately after 2000, are mentioned as base restrictions not the induced internal electric fields but the induced current densities that could produce stimuli of the central nervous system. These values were around $10 \mathrm{~mA} / \mathrm{m}^{2}$.

Anyway, the established current density $J\left[\mathrm{~A} / \mathrm{m}^{2}\right]$ and induced internal electric field $E_{\text {int }}[\mathrm{V} / \mathrm{m}]$ are vector related by the frequency dependent electric conductivity $\sigma[\mathrm{S} / \mathrm{m}]$ of the medium, equation (1), a customized expression of Ohm's law:

$J=\sigma E_{\text {int }}$

Initiating from these basic restrictions, reference levels (maximum accepted values for the incident, exterior electric field, also called ALs) have been set, which should not be exceeded.

The basic idea is that an accurate and non-invasive measurement of about $1 \mathrm{~V} / \mathrm{m}$ electric fields or $10 \mathrm{~mA} / \mathrm{m}^{2}$ current densities (all set inside the human body) represents a virtually impossible task. Measuring four orders of magnitude higher electric fields in the free space is a much more accessible assignment. These reference levels are calculated on the basis of simplified models, taking into account the maximum possible coupling of the field to the exposed person, representing a well desired safety margin.

Experts dealing with the dosimetry for exposure to LFEF, in principle agree with some general statements [9], [10]:

- considering a certain external field, the highest values of the internal electric field are registered when the body is firmly electrically grounded, while the lowest values are associated with the "free space" situation (body insulated from the ground);

- the current established in the grounded body is significantly influenced by physical dimensions (height and weight) and also by the posture (seated, standing or even lying on the floor) [11], [12];

- the distribution of the induced currents inside the body is not uniform, being decisively determined by the conductivity of various organs and tissues;

- the maximum coupling is met when the external electric field is parallel to the body vertical axis.

It is useful to remember that a contact with a good conductor placed in an electric field could be a source for indirect currents set up in the body.

As order of magnitude, the internal induced field is much smaller than the exterior one (for instance, about six times smaller at the power frequency). The internal electric field strength is very complicated to be assessed but could be computed by using electrically sophisticated heterogeneous models. It is advisable to apply a supplementary reduction factor of 3 to the so calculated values, in order to cover the great uncertainties in the dosimetric domain [13], [14].

At first glance, the field internally induced in the human body seems far too small compared to the outer electric field. This can be easily understood by imposing the continuity condition on the electric flux density, which is the product of the dielectric permittivity and the electric field strength. At low frequencies, the relative permittivity of human tissues and 
organs has values in the order of $10^{5}-10^{7}$ [15].

These action levels could be measured or calculated (analytical or by modeling), representing the highest values encountered at the employees' body position. We have to note that in specific non-sinusoidal conditions, the exposure evaluation must be based on the weighted peak method, involving filtering in the time domain, [16].

Two levels of exposure limits are to be considered:

- for general population, also called "public" or "residential" (people of various ages and state of health, including children and grey-headed persons, untrained and uninformed, being possible to stay 24 hours a day in that environment);

- for certain groups of workers (the so-called occupational exposure, in a controlled environment), for trained, informed, well-conditioned adults, working only a few hours per day in that restricted environment while respecting some protection rules.

In Table 2 there are summarized the reference values for the external E-field strength, accepted by ICNIRP [6].

The frequency $f$ included in these formulae is expressed in Hz.

The maximum permissible exposure (MPE) levels, the IEEE designation, corresponding to a certain degree to ICNIRP reference levels, for the human whole body exposure to sinusoidal electric fields in the background, stipulated in [8], are reported in Table 3. For occupational situations, IEEE utilizes the around synonymous "controlled environment". The ratio between occupational and public acceptable level is 4(frequencies up to $272 \mathrm{~Hz}$ ) and varies from 2 (ICNIRP recommendations) to 3 (IEEE restrictions) for the higher spectrum.

The European Directive 2013/35/EU is mainly

Table 2. ICNIRP reference levels (both occupational and public, rms values) for exposure to time varying electric fields.

\begin{tabular}{|c|c|c|}
\hline $\begin{array}{c}\text { Frequency range } \\
(\mathbf{H z})\end{array}$ & $\begin{array}{c}\text { E-field strength } \\
(\mathrm{V} / \mathrm{m}), \text { occupational } \\
\text { exposure }\end{array}$ & $\begin{array}{c}\text { E-field strength } \\
\mathbf{( V / m )} \\
\text { public exposure }\end{array}$ \\
\hline $1-50$ & 20.000 & 5.000 \\
\hline $50-3 \times 10^{3}$ & $5 \times 10^{5} / \mathrm{f}$ & $2.5 \times 10^{5} / \mathrm{f}$ \\
\hline $3 \times 10^{3}-10^{7}$ & 170 & 83 \\
\hline
\end{tabular}

Table 3. Maximum permissible exposure (MPE) to LFEF, $f$ is the frequency expressed in hertz $(\mathrm{Hz})$.

\begin{tabular}{|c|c|c|}
\hline $\begin{array}{c}\text { Frequency } \\
\text { range (Hz) }\end{array}$ & $\begin{array}{c}\mathrm{E}(\mathrm{V} / \mathrm{m})-\mathrm{rms}, \\
\text { controlled } \\
\text { environment }\end{array}$ & $\begin{array}{c}\mathrm{E}(\mathrm{V} / \mathrm{m})-\mathrm{rms}, \\
\text { general public, } \\
\text { (residential) }\end{array}$ \\
\hline $1-272$ & 20.000 & 5.000 \\
\hline $272-368$ & $5.44 \times 10^{6} / \mathrm{f}$ & 5.000 \\
\hline $368-3 \times 10^{3}$ & $5.44 \times 10^{6} / \mathrm{f}$ & $1.84 \times 10^{6} / \mathrm{f}$ \\
\hline $3 \times 10^{3}-10^{7}$ & 1810 & 610 \\
\hline
\end{tabular}

Table 4. Action levels (rms values) for occupational exposure, according to Directive 2013/35/EU/2013 ( $f$ expressed in Hz).

\begin{tabular}{|c|c|c|}
\hline $\begin{array}{c}\text { Frequency range } \\
(\mathbf{H z})\end{array}$ & $\begin{array}{c}\text { E-field strength } \\
\text { (V/m), Low ALs }\end{array}$ & $\begin{array}{c}\text { E-field strength } \\
\text { (V/m), High ALs }\end{array}$ \\
\hline $1-25$ & 20.000 & 20.000 \\
\hline $25-50$ & $5 \times 10^{5} / f$ & 20.000 \\
\hline $50-1640$ & $5 \times 10^{5} / f$ & $10^{6} / f$ \\
\hline $1640-3000$ & $5 \times 10^{5} / f$ & 610 \\
\hline $3000-10^{7}$ & 170 & 610 \\
\hline
\end{tabular}

concentrated on occupational exposure, with two levels of "alarm", low and high, presented in Table 4. While reaching them, some actions are strictly recommended, aiming to reduce the exposure, e.g. to increase the distance or to screen.

Anatomically realistic or simpler phantom models could be used for the calculation of induced current densities or induced fields, an example being the Maxwell surface-based phantom used in the practical guide associated with the Directive 2013/35/EU.

\section{MAIN SOURCES OF LFEF}

The most frequently encountered sources of LFEF (being significant even in terms of the power involved or due to their omnipresence in our daily environment) are:

- $\quad 50 \mathrm{~Hz}$, mains power transport and distribution system (North America and partly, Japan use the frequency of $60 \mathrm{~Hz}$ );

- the power supplied for traction systems (there is a certain variety, there are trams supplied at 600 Volts DC, electric locomotives in Romania are supplied with $50 \mathrm{~Hz}$ power while in Germany, Deutsche Bahn network uses power having the frequency of $16.7 \mathrm{~Hz}$ );

- electrical welding installations or other equipment requiring higher voltages, use working frequencies between 50 $\mathrm{Hz}$ and $200 \mathrm{~Hz}$;

- most power converters have operating frequency between $200 \mathrm{~Hz}$ and $1 \mathrm{kHz}$;

- older generation Cathode Ray Tube Monitors and TVs have $31 \mathrm{kHz}$ as operating frequency;

- the working frequency of economic lamps is varying in the range $40-45 \mathrm{kHz}$, depending on power and manufacturer;

- the working frequency of LCD monitors made in TFT (Thin Film Transistor) technology is in the range $42 \mathrm{kHz}-57$ $\mathrm{kHz}$ (depending on the manufacturer, but also on the diagonalsize);

switched mode power supplies, equipping PCs, laptops or printers have their working frequencies in the range 30 - $70 \mathrm{kHz}$

- $\quad$ ignition systems of injection engines;

- a specific type of radio-frequency service system (RFID), which uses electromagnetic fields to identify and automatically track a specific labeled object (which received a "tag" that contains electronically stored information), usually uses the $125-135 \mathrm{kHz}$ frequency range.

Anyway, the distance $d$ between the emitting source and the measuring point is essential. The electric field is inversely proportional to $d^{3}$ for high impedance sources, or only to $d^{2}$ for sources having low impedance (rough approximation).

\section{DETERMINATION OF THE EXPOSURE METRIC}

As we have seen, aiming to predict and to avoid health risks in epidemiology, some recommended exposure limits were established, associated with a frequency domain. The issue is very complicated as the electric field strength is a multidimensional quantity that varies over space and time, while its interaction with the human body is considerably influenced by the frequency.

The approach is even more challenging if the field polarization is non-linear, the sources have multiplefrequencies, the fields are non-uniform (spatially or temporally), non-sinusoidal or with high harmonic content. It is strongly desirable to have a single number summing up a certain 
exposure that should be compared with the Action Levels. Many measurements and an algorithm for data processing and analysis are necessary

In the low frequency domain (up to $100 \mathrm{kHz}$ ), the metrics with high significance are the peak vector magnitude or, mainly, the rms vector magnitude, averaged over the specified period.

In situations of simultaneous exposure to E-fields having different frequencies or non-sinusoidal fields, having significant harmonics, compliance with the imposed limits should be additively checked as stipulated in (2):

$\sum_{i=1 \mathrm{~Hz}}^{100 \mathrm{kHz}} \frac{E_{\text {imeas }}}{E_{i \text { Ref }}} \leq 1$

where $E_{\text {imeas }}$ represents the rms values of electric field measured for every frequency of the spectrum and $E_{\mathrm{iRef}}$ is the corresponding reference value for that frequency, as it is specified in the appropriate Table 2, Table 3 or Table 4.

Aiming to decompose the fields with complex, pulsed patterns, the Fast Fourier Transformation techniques are the best option, usually included in the firm software of now-a-days (even hand-held) spectrum analyzers.

\section{ASSESSING THE EXPOSURE TO LFEF IN A LABORATORY CLASS WITH 16 NETWORKED PCS.}

As basic device we have used a hand-held, low frequency spectrum analyzer, SPECTRAN NF 5305, by USB connected to a laptop running the MCS dedicated software produced by Aaronia AG Electronics Manufacturer, Germany. Even if this spectrum analyzer already has an internally integrated sensor for electric fields, we have complementary used an external capacitive sensor, battery powered, connected at the provided external input (SMA socket) of the device, Figure 1 [17].

Unlike the case of close magnetic field sensors, the physical detection principles for low frequency electric fields are less numerous. Very important is the influence that the sensor itself exerts on the detected field strength, the frequency related sensitivity, detection range and linearity. A modern approach for electric field measuring should be the electro-optical effect or the variability of gate to source voltage for some special field effect transistors.

Quasi affordable for manufacturing in an ordinary laboratory is the measurement of induced electrical charges on a sensing capacitor (with magnitude order of 100-200 pF), followed by a differential amplifier.

The sensor's geometry could be plate (rectangular) or even spherical. The induced charge on one half of this field meter is directly proportional to the electric field that is parallel to its axis, the sensitivity depending on the effective area where the electrical charge could be induced:

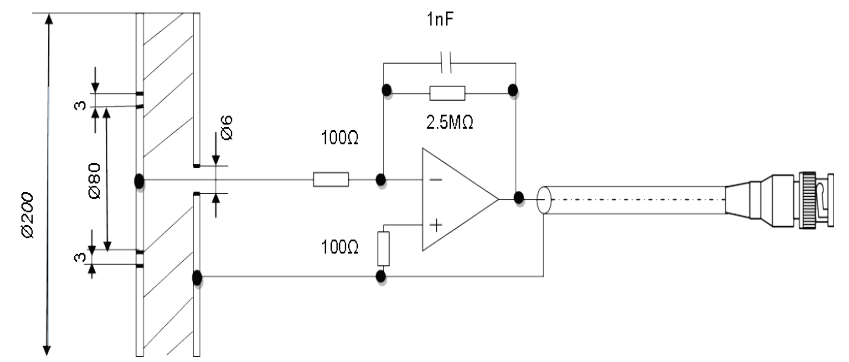

Figure 1. Capacitive E-field sensor made from PCB double layer, $2 \mathrm{~mm}$ thickness.
$Q=S \varepsilon_{0} E$

where, as we have previously stated, $S$ is proportional to the sensor's active surface area, in our case $50 \mathrm{~cm}^{2}$ (the surface of a circle with $4 \mathrm{~cm}$ radius).

The current established between the electrodes of the capacitive sensor is the time derivative of the induced charge (the field being time-harmonic) while the meter is considered as "free body" (isolated from the ground):

$I=j \omega \mathrm{S} \varepsilon_{0} E$

Another type of sensor, this time non-directional that can also be easily hand-crafted, is presented in Figure 2.

Due to the relatively small length (and surface), this sample has little sensitivity compensated by the advantage of accurately locating the emitting source.

If a more sensitive sample is desired (which can "pick" lower value fields) at the cost of reducing the resolution, the key might be the "sphere" sample. The handle is made of a semirigid coaxial cable of $50 \Omega$ connected to the BNC connector by means of a resistor of $50 \Omega$ as impedance adaptation. At the other end of the coaxial cable, the central conductor is attached to a conductive sphere having a diameter of $3-5 \mathrm{~cm}$. The assembly becomes rigid due to the plastic insulator material, also preventing short circuits that might occur during measurements. Due to the presented spatial symmetry, both stub and sphere probes are omnidirectional.

With the hand-held spectrum analyzer, using its own E-field sensor or coupled with our hand-crafted ones, we have traced the profiles of the electric field strength in the laboratory class, the contours and a 3D map.

We proposed to measure the exposure around the regions where the heads of the 15 students are expected to be placed. The electric field could be easily perturbed by any (conductive) object; consequently, the classroom was without students, the sensor was mechanically introduced in the area of interest by using a 1.5 meter fiberglass extension pole and the PCs were running a repetitive routine.

At the first survey, E-fields having three frequencies were encountered: $50 \mathrm{~Hz}$ (the mains power), $56 \mathrm{kHz}$ (working frequencies of the 16 TFT-LCD displays) and $62 \mathrm{kHz}$ (much

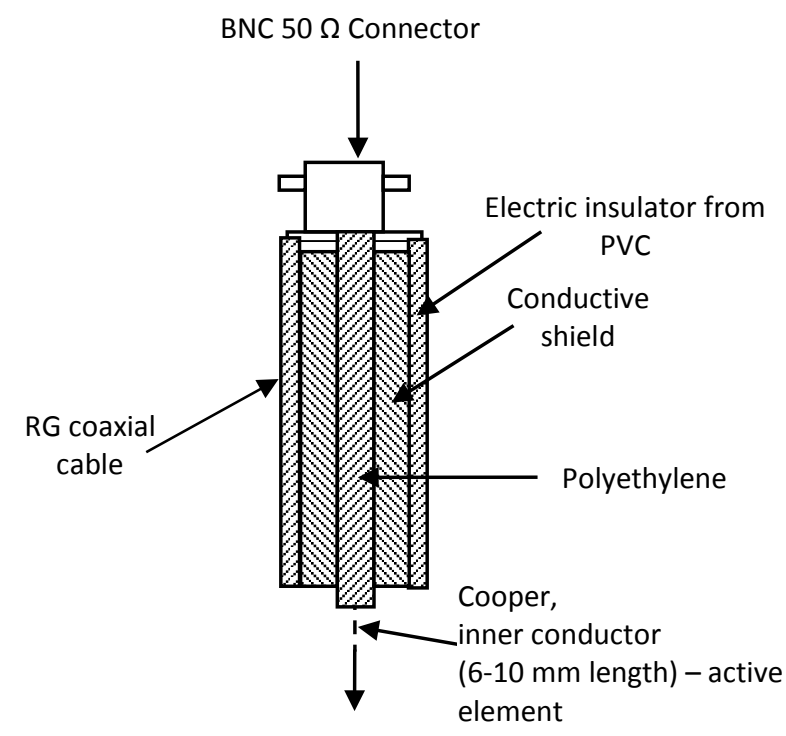

Figure 2. Stub type, low sensitivity but omnidirectional, near electric field probe. 
lower values than the previous ones, being associated with the Switched Mode Power Supply of the PCs, easy to be shielded).

The rms readings were averaged over space and time (5 minutes).

These values, expressed in $\mathrm{V} / \mathrm{m}$, for the 16 places of interest and 2 significant frequencies are shown in Figure 3.

Their spatial distribution in the laboratory room is presented in Figure $4 \mathrm{a}$ (for $50 \mathrm{~Hz}$ ) and Figure $4 \mathrm{~b}$ (for $56 \mathrm{kHz}$ ).

We have applied (2) as the sum of ratios between the highest E-field values measured in the laboratory class, at $50 \mathrm{~Hz}, 56$ $\mathrm{kHz}$ and $62 \mathrm{kHz}$ and the corresponding reference levels accepted for public exposure:

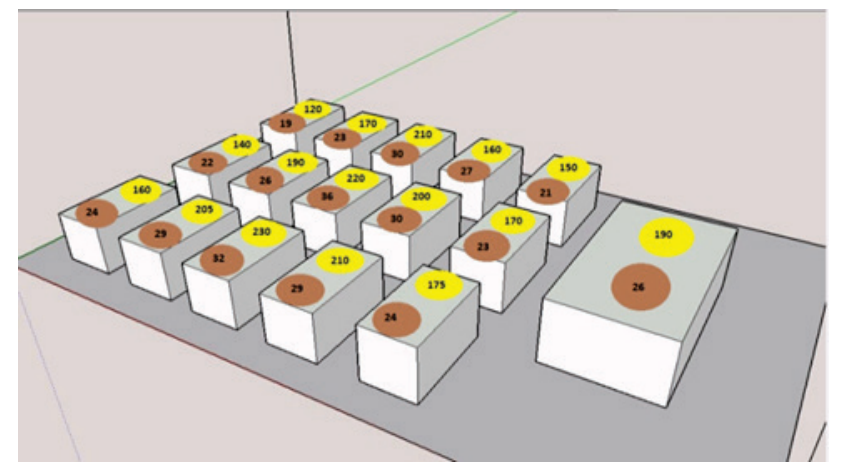

Figure 3. The measured values of the E-field strength, at $50 \mathrm{~Hz}$ (light yellow) and $56 \mathrm{kHz}$ (dark brown).

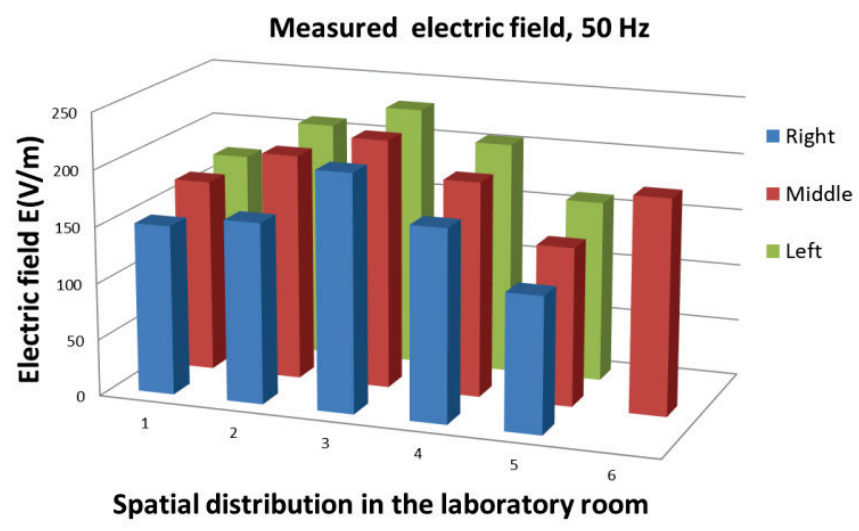

Figure 4a. The 3D map of the $50 \mathrm{~Hz}$ E-fields.

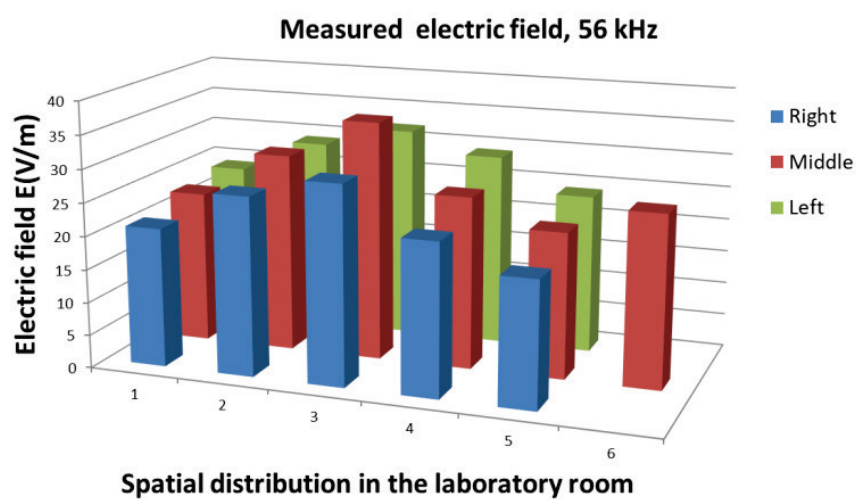

Figure $4 \mathrm{~b}$. The $3 \mathrm{D}$ map of the $56 \mathrm{kHz}$ E-fields. $\sum_{i=50 \mathrm{~Hz}}^{62 \mathrm{kHz}} \frac{E_{\text {imeas }}}{E_{\text {Ref }}}=\frac{230}{5000}+\frac{36}{83}+\frac{9}{83}=0.588 \leq 1$

This value is significantly below the accepted unit value, even for public, non-occupational exposure.

\section{7. “IN SILICO" MEASUREMENT OF THE ELECTRIC FIELD PRODUCED BY OVERHEAD POWER LINES}

The overhead power lines (OPLs) are major sources of LFEF which might reach, in specific conditions, the Action Levels stated by Directive 2013/35/EU. We have performed direct measurements with the device and the external sensor previously described for various High Voltage OPLs. The registered values were used for checking and validation of the proposed numerical method. In the scientific literature, there are many papers dealing with the computation of LFEF produced by overhead power lines, e.g. [18]-[23], employing both analytical and numerical methods. We developed here a simple numerical approach based on Finite Element Method Magnetics 4.2 (FEMM 4.2) [24], which is open source finite element software for solving low frequency electromagnetic problems on two-dimensional planar and axisymmetric domains.

As can be seen from the very recent data literature, concerns about the calculation of electric fields produced by overhead power lines are still topical. There are either variants of using very powerful general software [25], alongside specific methods (such as charge simulation method, CSM) implemented in general mathematical computation software [26] or using simpler, 2D or more elaborate 3D models [27].

This domain is also very topical from the reverse perspective, by developing reliable and cost-effective noncontact monitoring techniques for high voltage transport and distribution networks [28], [29].

In a previous study [30], we have also applied FEMM 4.2 to compute low frequency magnetic fields associated with common configurations of OPLs used in Romanian power transport and distribution systems.

\subsection{Double-circuit power line and associated FEMM model}

OPL selected for this case study is a typical $110 \mathrm{kV}$ doublecircuit line (DCL) with the xy coordinates of the 7 conductors presented in Table 5.The architecture is determined by the steel pylons Sn 110.252 type, [31]. The active $2 \times 3$ Aluminum Conductor Steel Reinforced (ACSR) conductors have a crosssection $240 / 40 \mathrm{~mm}^{2}$ (core diameter $8.1 \mathrm{~mm}$ and exterior diameter $21.70 \mathrm{~mm}$ ). The ACSR ground wire has across-section $160 / 95 \mathrm{~mm}^{2}$ (core diameter $12.75 \mathrm{~mm}$ and exterior diameter $20.75 \mathrm{~mm}$ ). The sag effect is taken into consideration by specifying three ground clearances, namely $h=8 \mathrm{~m}, \mathrm{~h}=12 \mathrm{~m}$

Table 5. Input data for the considered $110 \mathrm{kV}$ double-circuit line.

\begin{tabular}{|c|c|c|c|}
\hline Conductor & $\mathbf{i}$ & $\mathbf{x}_{\mathbf{i}}(\mathbf{m})$ & $\mathbf{y}_{\mathbf{i}}(\mathbf{m})$ \\
\hline \multirow{2}{*}{ Phase I, $110 / \sqrt{ } 3 \mathrm{e}^{\mathrm{j} 0^{\circ}}$} & 1 & -3.05 & \multirow{2}{*}{$\mathrm{h}$} \\
\cline { 2 - 3 } & 2 & 3.05 & \\
\hline \multirow{2}{*}{ Phase II, $110 / \sqrt{ } 3 \mathrm{e}^{\mathrm{j} 240^{\circ}}$} & 3 & -5.00 & \multirow{2}{*}{$\mathrm{h}+4.60$} \\
\cline { 2 - 3 } & 4 & 5.00 & \\
\hline \multirow{2}{*}{ Phase III, $110 / \sqrt{ } 3 \mathrm{e}^{\mathrm{j} 120^{\circ}}$} & 5 & -3.05 & \multirow{2}{*}{$\mathrm{h}+10.30$} \\
\cline { 2 - 3 } & 6 & 3.05 & \\
\hline Ground wire, 0 & 7 & 0.00 & $\mathrm{~h}+16.25$ \\
\hline
\end{tabular}


and $\mathrm{h}=15 \mathrm{~m}$.

The power line FEMM model is shown in Figure 5a, meshed by a net of 252900 nodes and 505375 triangular elements.

The boundary conditions here applied assume that the scalar electric potential at the ground level and along the semi-circular boundary is zero $(U=0)$. The air region surrounding the conductors is taken large enough to neglect the side effects, having a radius of at least 10 times the arrangement dimensions.

All conductors are modeled as presented in Figure 5b, using the following electric parameters: for aluminum (Al), $\sigma=$ $3.77 \times 10^{7} \mathrm{~S} / \mathrm{m}$ and $\varepsilon_{\mathrm{r}}=1$; for steel $(\mathrm{Ol}$, internal symbol), $\sigma=$ $0.56 \times 10^{7} \mathrm{~S} / \mathrm{m}$ and $\varepsilon_{\mathrm{r}}=1$. The analysis type is set to "current flow" ( $f=50 \mathrm{~Hz}$ ), which generally allows to determine electric field distributions, voltage distributions, current densities, etc.

\subsection{Electric field simulation results}

Figure 6 compares lateral profiles of the rms electric field strength calculated at the standard height of $1 \mathrm{~m}$ above the ground for the aforementioned ground clearances. For $h=8 \mathrm{~m}$, a relatively low clearance, the maximum electric field strength under the power line is $2.18 \mathrm{kV} / \mathrm{m}$, which is 2.3 times below de

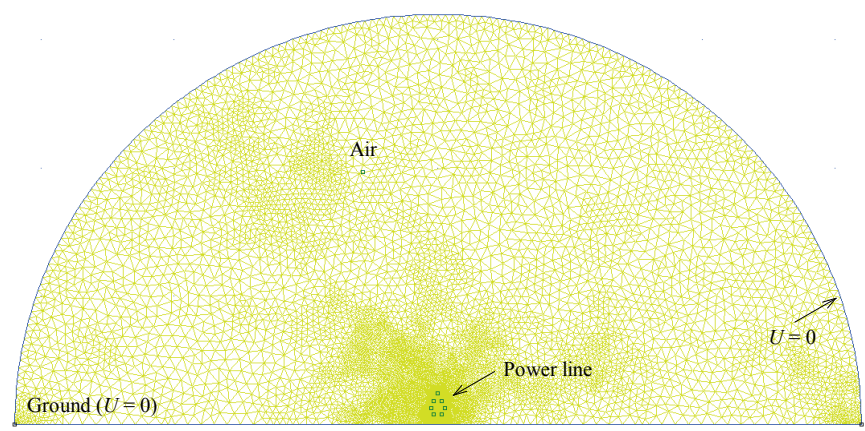

Figure 5a. FEMM 4.2 model for a $110 \mathrm{kV}$ double-circuit power line: meshed global model.

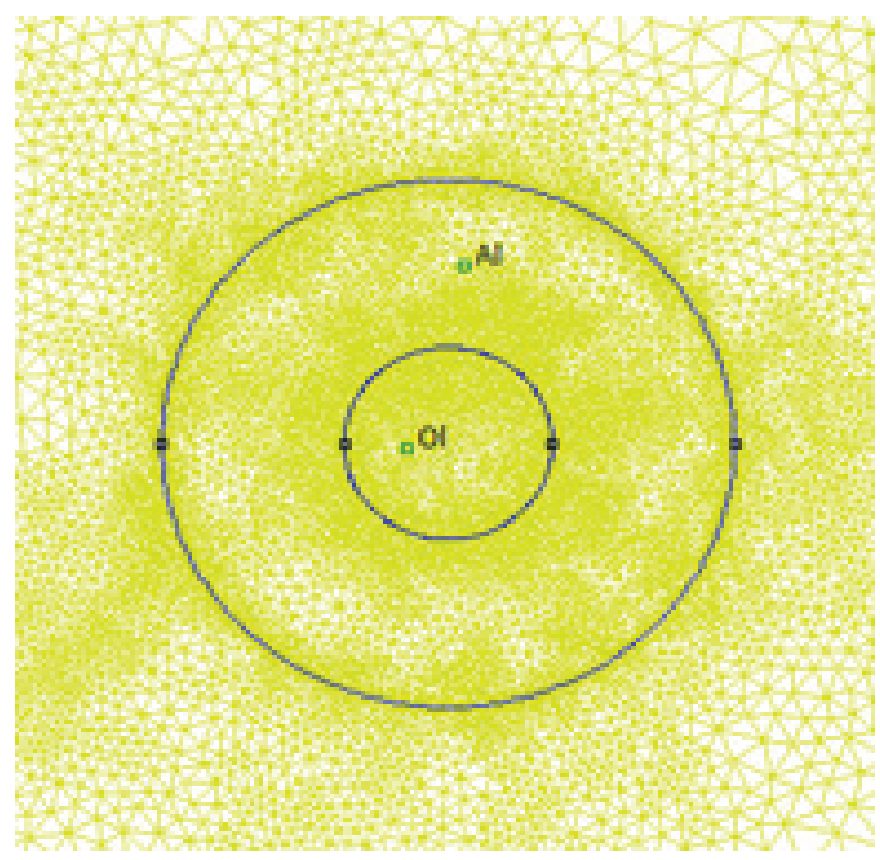

Figure 5b. FEMM 4.2 model for a $110 \mathrm{kV}$ double-circuit power line (conductor's cross-section details).

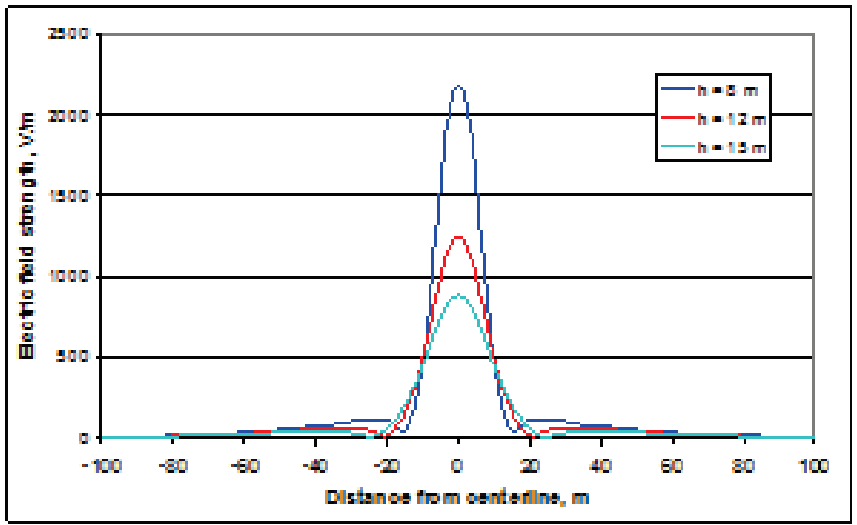

Figure 6. Lateral profiles of the rms electric field strength from the considered $110 \mathrm{kV} \mathrm{DCL}$, calculated for a height of $1 \mathrm{~m}$ above the ground.

EU reference (public) level, $5 \mathrm{kV} / \mathrm{m}$. At $18.5 \mathrm{~m}$ distance from the centerline, which represents the semi-width of the safety / protection zone for $110 \mathrm{kV}$ overhead high-voltage lines (according to the national regulations) [31], the electric field strength is much lower, about $90 \mathrm{~V} / \mathrm{m}$. For $h=12 \mathrm{~m}$, an intermediate clearance, the maximum electric field strength under the power line is $1.25 \mathrm{kV} / \mathrm{m}$, while at $18.5 \mathrm{~m}$ distance from the centerline the field strength falls below $50 \mathrm{~V} / \mathrm{m}$.

The distribution of the electric field around the power line is presented in Figure $7(h=8 \mathrm{~m})$, where the horizontal contour indicates the width of the safety zone.

As it can be concluded from both Figure 6 and Figure 7, the electric field exposure levels outside this area are very low, fast decreasing with distance. Note that the E-field density plot in Figure 7 is given in terms of peak value, as directly generated by FEMM 4.2.

Results obtained with this FEMM approach have been compared with similar calculations carried out with software tools based on quasi-static analytical methods [20], as well as with measurements conducted in vicinity of several power lines. All the approaches lead to approximately comparable results.

\section{CONCLUSIONS AND FURTHER ATTEMPTS}

EU Member States have currently transposed Directive $35 / 2013 / \mathrm{EU}$ into their legislation. It is necessary to provide, both for the employers and for the appropriate authorities, an applicable procedure, based on a simple metric and budget

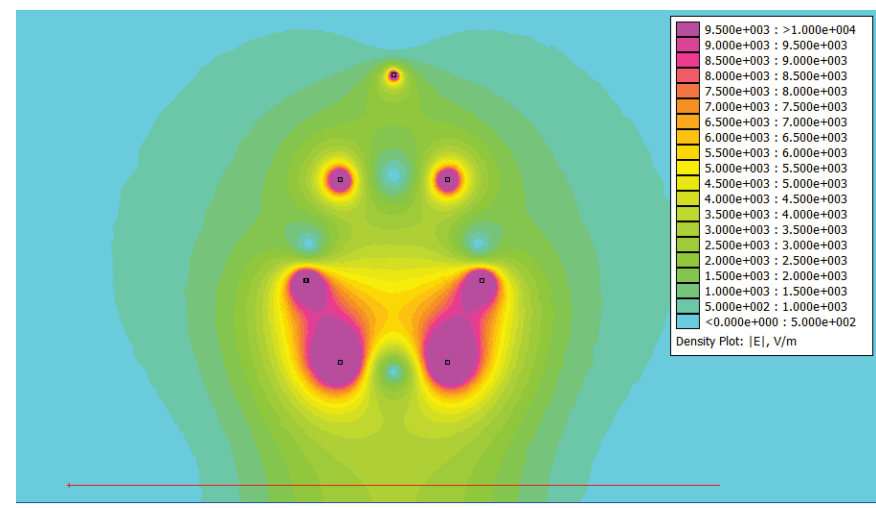

Figure 7. Electric field distribution around the $110 \mathrm{kV} \mathrm{DCL}(h=8 \mathrm{~m})$. 
equipment, aiming to decide by measurements, if a specific location is risky or not from the point of view of the exposure to electric (and also magnetic) fields. We have presented the approach intended to deal this question only for LFEF, based on an affordable handheld spectrum analyzer, easy to be made additional sensors and a cumulative metric derived from the rms vector magnitude.

The paper also stimulates the use of free-open source software, which can model complex configurations from the point of view of the generated electric (or magnetic) fields. To avoid systematic errors, it is advisable to verify the comparability between directly measured values and those obtained by modeling and simulation.

These domestic tests have the role of inexpensive internal control being namely oriented to small businesses. If there are found values close to the maximum allowed ones, it could be decided on the opportunity of calling for specialized firms.

As a further concern, we want to present a modeling proposal for the current densities simultaneously induced in the human body by the electric and magnetic fields. Concretely, through the HV-OPLs presented in the paper, usually pass currents of hundreds of amperes, so the generated magnetic field is also considerable. If the currents induced in the human body by the electric field produced by these HV-OPLs are vertical and linear, the ones produced by the magnetic field are shaped like circular loops. There might be an exposure risk in certain organs (e.g. one lung or one kidney). It is possible as the overlapping of these currents to be cumulative-additive, a matter that should need to be taken into account both in modeling and in legal regulations.

\section{REFERENCES}

[1] European Commission, "Directive 2013/35/EU of the European Parliament and of the council of 26 June 2013 on the minimum health and safety requirements regarding the exposure of workers to the risks arising from physical agents (electromagnetic fields)", Official J. Eur. Union, 2013, 1791, pp. $1-21$.

[2] European Commission, "Non-binding guide to good practice in implementing Directive 2013/35/EU - Electromagnetic Fields, Volume 1: Practical Guide", 2015

[3] European Agency for Safety and Health at Work, "European survey of enterprises on new and emerging risks", https://osha.europa.eu/en/node/6745/file_view.

[4] M. Morega, I. M. Baran, A. M. Morega, "Evaluation of environmental low frequency magnetic fields in occupational exposure", Proc. of 8th Int. Conf. and Exposition on Electrical and Power Engineering EPE-2014, Oct. 16-18, 2014, Iasi, Romania, pp. 522-527.

[5] ICNIRP, "Guidelines for limiting exposure to time-varying electric, magnetic and electromagnetic fields (up to $300 \mathrm{GHz}$ )", Health Physics 74(4), 1998, pp.494-522.

[6] ICNIRP, "Guidelines for limiting exposure to time-varying electric and magnetic fields $(1 \mathrm{~Hz}-100 \mathrm{kHz}) "$, Health Physics 99(6), 2010, pp.818-836.

[7] IEEE-ICES Standard C95.6-2002, "Standard for safety levels with respect to human exposure to electromagnetic fields, 0-3 $\mathrm{kHz} ", 2002$.

[8] IEEE-ICES Standard C95.3.1-2010, "IEEE recommended practice for measurements and computations of electric, magnetic, and electromagnetic fields with respect to human exposure to such fields, $0 \mathrm{~Hz}$ to $100 \mathrm{kHz} ", 2010$.

[9] J.D. Bowman, M.A. Kelsh, W.T. Kaune, Manual for measuring occupational electric and magnetic field exposures, DHHS (NIOSH) Publication No. 98-154, 1998.
[10] V. De Santis, X.L. Chen, "On the issues related to compliance assessment of ICNIRP 2010 basic restrictions" Journal of Radiological Protection, 2014, 34 (2), pp. N31-N39.

[11] P. Dimbylow, R. Findlay, "The effects of body posture, anatomy, age and pregnancy on the calculation of induced current densities at $50 \mathrm{~Hz} . "$, Journal of Radiation protection dosimetry, 2010, 139 (4), pp. 532-538.

[12] R.P. Findlay, "The effects of human height and mass on the calculated induced electric fields at $50 \mathrm{~Hz}$ for comparison with the EMF Directive 2013/35/EU", Journal of Radiological Protection 2017, Volume 37, Issue 1, pp. 201-213.

[13] ISO/IEC Guide 98-3:2008, "Uncertainty of measurement-Part 3: Guide to the expression of uncertainty in measurement", 2008.

[14] S.W.Park, K. Wake, S. Watanabe, "Calculation errors of the electric field induced in a human body under quasi-static approximation conditions", IEEE Transactions on microwave theory and techniques, 2013, Volume 61, Issue 5, pp. 2153-2160.

[15] C.Gabriel, S. Gabriel, E.Courthout, "The dielectric properties of biological tissues, I. Literature survey", Physics in Medicine\&Biology, 1996, 41, pp.2231-2249.

[16] ICNIRP, "ICNIRP statement-guidance on determining compliance of exposure to pulsed and complex non-sinusoidal waveforms below $100 \mathrm{kHz}$ with ICNIRP guidelines", Health Physics, 2003, vol. 84, no. 3, pp. 383-387.

[17] A. Salceanu, M.M. Poenaru, M.A. Anghel, M. Paulet, "Approach on the evaluation of exposure to low frequency electric fields", Proc. of 21st IMEKO TC4 International Symposium and 19th International Workshop on ADC Modelling and Testing, Sept. $7-$ 9, 2016, Budapest, Hungary, pp. 32-36.

[18] B. Ali Rachedi, A. Babouri, F. Berrouk, "A study of electromagnetic field generated by high voltage lines using COMSOL MULTIPHYSICS", Proc. of 2014 International Conference on Electrical Sciences and Technologies in Maghreb (CISTEM), Nov. 3-6, 2014, Tunis, Tunisia, pp. 1-5.

[19] L. Xiao, K.E. Holbert, "Development of software for calculating electromagnetic fields near power lines", North American Power Symp. (NAPS), Sep. 7-9, 2014, Pullman, U.S., pp. 1-6.

[20] S.S. Razavipour, M. Jahangiri, H. Sadeghipoor, "Electrical field around the overhead transmission lines", World Academy of Science, Engineering and Technology 2012, 6, pp. 168-171.

[21] H. Ahmadi, S. Mohseni, A.A. ShayeganiAkmal, "Electromagnetic fields near transmission lines - problems and solutions", Iran. J. Environ. Health. Sci. Eng. 2010, 7, pp. 181-188.

[22] M. Milutinov, A. Juhas, M. Prša, "Electromagnetic field underneath overhead high voltage power line", Proc. of 4th International Conference on Engineering Technologies, April 2830, 2009, Novi Sad, Serbia, pp. 1-5.

[23] S. Vujević, D. Lovrić, T. Modrić, "2D computation and measurement of electric and magnetic fields of overhead electric power lines", Proc. of the Joint INDS'11 \& ISTET'11, July 2527, 2011, Klagenfurt, Austria, pp. 1-6.

[24] D. Meeker, "Finite Element Method Magnetics, Version 4.2, User's Manual", October 25, 2015, http://www.femm.info/Archives/doc/manual42.pdf.

[25] I.N.Ztoupis, I.F.Gonos, I.A.Stathopulos, "Measurement and calculation of power frequency electric fields generated by high voltage overhead power lines", Proc. of International Conference on High Voltage Engineering and Application (ICHVE), September 08-11, 2014, Poznan, Poland.

[26] A. Rankovic, "Novel multi-objective optimization method of electric and magnetic field emissions from double-circuit overhead power line", International Transactions On Electrical Energy Systems, 2017, Volume 27, Issue 2.

[27] T. Modric, S. Vujevic, "Computation on the electric field in the vicinity of overhead power line towers", Electric Power Systems Research, 2016, Volume 135, pp. 68-76.

[28] K. Zhu, W. K. Lee, W.T. Philip Pong, "Non-contact capacitivecoupling-based and magnetic-field-sensing-assisted technique for monitoring voltage of overhead power transmission lines", IEEE Sensors Journal, 2017, Volume 17, Issue 4, pp. 1069-1083. 
[29] M.A. Noras, "Electric and magnetic field monitoring for power line diagnostics", Proc. of 51-st Annual Meeting of the IEEEIndustry Applications Society, October 11-22, 2015, Addison.

[30] E. Lunca, M. Istrate, A. Salceanu, "Comparative analysis of the extremely low-frequency magnetic field exposure from overhead power lines", Environmental Engineering and Management Journal, 2013, 12, pp. 1145-1152.
[31] ANRE, NTE 003/04/00 - "Normative document regarding the construction of overhead electric lines exceeding $\mathrm{AC} 1 \mathrm{kV}$ " (in Romanian), 2014, URL:

http://www.anre.ro/ro/legislatie/documente-de-discutieee1/regl-tehnice-regulamente/normativ-pentru-constructialiniilor-aeriene-de-energie-electrica-cu-tensiuni-peste$1000 \mathrm{v} \&$ page $=2$. 